\title{
Development of Next Generation Sequencing panel including cancer risk gene and drug-metabolizing enzymes
}

\author{
Phuong T. T Nguyen ${ }^{1}$, Hye-Eun Jeong ${ }^{1}$, Ho-Sook Kim ${ }^{1,2}$, Jong-Lyul Ghim ${ }^{1,2}$, Jae Gook Shin ${ }^{1,2}$ \\ ${ }^{I}$ Department of Pharmacology and PharmacoGenomics Research Center, Inje University College of Medicine, Busan, \\ Korea, ${ }^{2}$ Department of Clinical Pharmacology, Inje University Busan Paik Hospital, Busan, Korea
}

BACKGROUND: Somatic and hereditary germline mutations to increase the susceptibility to cancers have increased risk for developing cancer. Detection of these mutations in cancer patients can provide great benefits for diagnostics, prediction of prognosis and precision medicine. The objective of this study was to to develope and validated a custom Next Generation Sequencing (NGS) panel for analysis of known cancer-related biomarker.

METHODS: Panel was designed a multiplex PCR targeting hotspot region of 20 genes (AKT1, BRAF, EGFR, ERBB2, FOXL2, KIT, KRAS, MET, BRCA1, BRCA2, NRAS, PDGFRA, PIK3CA, RET, TP53, UGT1A1, CYP2D6, TPMT, MTHFR and DPYD) which are for cancer risk assessment, and selection of drug and drug dosage. We performed an analytical validation on 20 samples including 10 cell lines and 10 formalin-fixed paraffin-embedded samples.

RESULTS: Validation revealed sensitivity and specificity of $100 \%$ for detection of mutations compared with conventional Sanger sequencing and TruSight ${ }^{\circledR}$ Tumor 15 panel.

CONCLUSION: Our custom cancer panel will provide an accurate, convenient, and fast genotyping method to detect multiple variants with reduced time and cost of genetic analysis allowing for strategic patient management. 